This is an electronic reprint of the original article. This reprint may differ from the original in pagination and typographic detail.

\author{
Author(s): Hokkanen, Matti; Lautala, Saara; Shao, Dongkai; Turpeinen, Tuomas; Koivistoinen, \\ Juha; Ahlskog, Markus
}

Title: On-chip purification via liquid immersion of arc-discharge synthesized multiwalled carbon nanotubes

Year: $\quad 2016$

Version:

Please cite the original version:

Hokkanen, M., Lautala, S., Shao, D., Turpeinen, T., Koivistoinen, J., \& Ahlskog, M. (2016). On-chip purification via liquid immersion of arc-discharge synthesized multiwalled carbon nanotubes. Applied Physics A: materials science \& processing, 122(7), Article 634. https://doi.org/10.1007/s00339-016-0154-0

All material supplied via JYX is protected by copyright and other intellectual property rights, and duplication or sale of all or part of any of the repository collections is not permitted, except that material may be duplicated by you for your research use or educational purposes in electronic or print form. You must obtain permission for any other use. Electronic or print copies may not be offered, whether for sale or otherwise to anyone who is not an authorised user. 


\title{
On-chip purification via liquid immersion of arc-discharge synthesized multiwalled carbon nanotubes
}

\author{
Matti J. Hokkanen - Saara Lautala - Shao Dongkai • \\ Tuomas Turpeinen • Juha Koivistoinen • Markus Ahlskog
}

Received: date / Accepted: date

\begin{abstract}
Arc-discharge synthesized multiwall carbon nanotubes (AD-MWNT) have been proven to be of high quality, but their use is very limited due to difficulties in obtaining them in a clean and undamaged form. Here we present a simple method that purifies raw AD-MWNT material in laboratory scale without damage, and that in principle can be scaled up. The method consists of depositing raw AD-MWNT material on a flat substrate and immersing the substrate slowly in water, whereby the surface tension force of the liquid-substrate contact line selectively sweeps away the larger amorphous carbon debris and leaves relatively clean MWNTs on the substrate. We demonstrate the utility of the method by preparing clean individual MWNTs for measurement of their Raman spectra. The spectra exhibit the characteristics of high quality tubes free from contaminants. We also show how one concomitantly with the purification process can obtain large numbers of clean suspended MWNTs.
\end{abstract}

Keywords Carbon nanotube - Multiwalled carbon nanotube $\cdot$ Particle detachment $\cdot$ Purification - Surface tension

M. J. Hokkanen · S. Lautala · S. Dongkai

University of Jyvaskyla, Department of Physics \&

Nanoscience Center.

T. Turpeinen

University of Jyvaskyla, Department of Physics.

J. Koivistoinen

University of Jyvaskyla, Department of Chemistry.

M. Ahlskog

University of Jyvaskyla, Department of Physics \&

Nanoscience Center.

P. O. Box 35, FI-40014 University of Jyvaskyla, Finland.

Tel.: +358 40805 4056, E-mail: markus.e.ahlskog@jyu.fi .

\section{Introduction}

The CVD-growth technique of multiwall carbon nanotubes (MWNTs) is presently the only viable option in applications that aim for mass production [1], since the alternative, the arc-discharge synthesized MWNTs (AD-MWNT), can not be produced in scaled up quantities in pure form. This state-of-affairs is unfortunate, since there is multiple experimental evidence that $\mathrm{AD}$ MWNTs are superior to CVD-grown on individual comparison with respect to physical properties. In electronic transport, a low level of defects enables a large mean free path, and thus ballistic transport. Quasiballistic transport has been observed only in the arcdischarge grown MWNTs [2,3], while in CVD-grown tubes conduction has been found to be close to strong localization [4]. Nanomechanical properties have also been directly compared in measurements [5], where the Youngs modulus in CVD-MWNTs was found greatly inferior compared to that of AD-MWNTs. In addition, sophisticated experiments have been reported that rely on the multilayered structure of MWNTs. Examples are telescopic extraction of the inner layers [6] and unzipping of the outer layer to form a graphene nanoribbon [7]. These fully depend on the quality and integrity of the tube layers as concentric SWNTs, and both have been performed with AD-MWNTs.

Purification and quality issues are a more pressing topic with MWNTs than with single wall carbon nanotubes (SWNT). Here "quality" refers to the quality of the tube itself, while "purity" refers to the extent to which the macroscopic MWNT material contains matter besides the tubes. The two synthesis techniques (CVD and $\mathrm{AD}$ ) are very different with respect to these issues. The AD technique produces tubes with uniform thickness and absolute straightness. Usually 
CVD-grown MWNTs have intrinsically curved shape, which is seen to stem from the defectiveness although no comprehensive experimental investigation regarding to the structural features of MWNTs has been performed. The main shortcoming of AD-MWNT material is that it contains excessively amorphous carbon in particles of all size ranges in the nanometer and micrometer domains. Various purification procedures have been employed [9], which have included filtration and treatment in concentrated acids. All of these have problems in that the quality of the tubes degrades significantly as mechanical or chemical damage occurs. Most of the sophisticated experiments mentioned above have been done with unpurified material as the starting point, from which the high quality tubes have been separated, often in labour intensive procedures. This state-of-affairs has limited the repeatability and impact of these works and therefore the perceived utility of AD-MWNTs, even in research settings.

In a very recent work [9], we found that the advancing contact line of a water droplet, placed on a thin deposition of AD-MWNTs, can separate the amorphous carbon debris from a good share of the MWNTs. In this work, we present a purification procedure for ADMWNT material that utilizes this effect on larger deposition areas. We obtain relatively clean MWNT deposits on $\mathrm{Si}$ substrates, in a process that involves practically no mechanical or chemical damage to the tubes. Furthermore, we present two concrete examples of how the process can be applied to prepare single AD-MWNTs for experiments. In the first we demonstrate Raman spectroscopy of clean individual AD-MWNTs, of which there hitherto exists very little data. In the second example we demonstrate how one concomitantly with the purification process can obtain large numbers of clean suspended MWNTs.

\section{Experimental}

The steps of the sample preparation and the experiment, are presented schematically in Fig. 1. MWNT material, consisting of both AD-MWNTs and irregular amorphous carbon debris was dispersed in 1,2-dichloroethane as-received without addition of dispersing agents. The dispersions were prepared in a relatively thick concentration $(0.25 \mathrm{mg} / \mathrm{ml})$ to obtain dense MWNT depositions. Cleaned Si substrates were made hydrophilic in oxygen plasma with reactive ion etching (RIE). MWNTs were deposited by spin coating a few drops of dispersion on the freshly treated surfaces. A typical scanning electron microscope (SEM) image of the resulting surface is shown in Fig. 3a. To allow for atomic force microscopy (AFM) imaging of

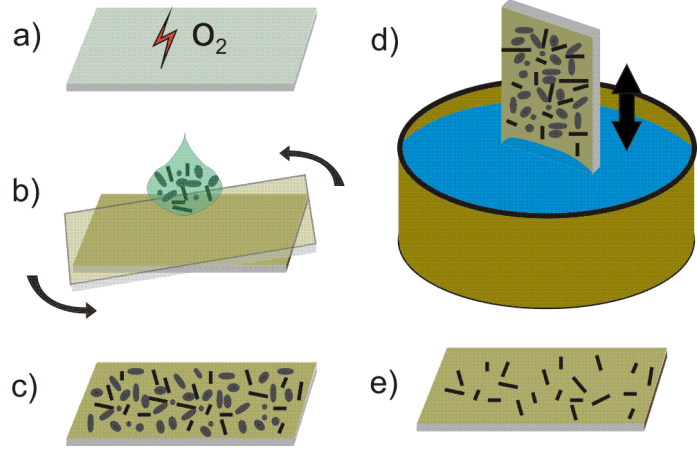

Fig. 1 Schematic of our purification process. a) Silicon chip made hydrophilic with RIE b-c) Deposition with spin coating of AD-MWNT material d) The immersion step: the liquid interface at the contact line sweeps over the chip. e) Si chip with purified MWNT deposition.

the same site prior to and following the immersion process, reference patterns were fabricated with electron beam lithography. The preparation of the patterned substrates for the experiments was similar to the nonpatterned ones.

The immersion experiments were carried out within 6 hours from the sample preparation to minimize the decay of surface chemistry [10]. The experimental setup is similar as in Refs. $[11,12]$, and consists of a motordriven three-axis stage controlled via computer interface for positioning and immersion of the sample suspended from its edge. The sample was immersed vertically (Figs. 1, 2b), into a test tube with ultrapure water, whereby the contact line (the air-water-substrate triple phase contact line) swept over the surface with a pre-determined velocity. The sample was lowered at constant immersion velocity $v_{\text {im }}$, varied from 0.01 to 1 $\mathrm{mm} / \mathrm{s}$, until it was completely submerged. A CCD camera was used for visualization of the immersion process.

It was found important to remove with a pipette several $\mathrm{ml}$ of water near the surface, as the detached particulate matter residing on the water surface, forming large rafts, had a tendency to become re-deposited onto the sample surface during the retraction phase. Despite this precaution, smaller re-deposited features are occasionally observed on the samples. Following the pipetting of the interface layer, the sample was quickly retracted $\left(v_{\text {re }} \sim 1 \mathrm{~mm} / \mathrm{s}\right)$, and dried with nitrogen gas.

The surfaces were imaged both before and after the immersion process with dark-field optical microscopy, SEM, and AFM. To evaluate the immersion process in an exact manner, AFM imaging was performed on the same locations both before and after the process, on samples with microfabricated reference grids. Also, the immersion velocity was varied between the different 


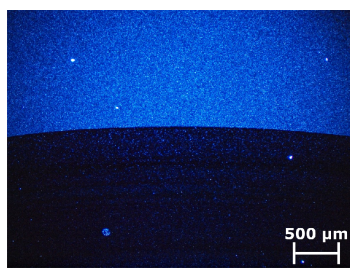

(a)

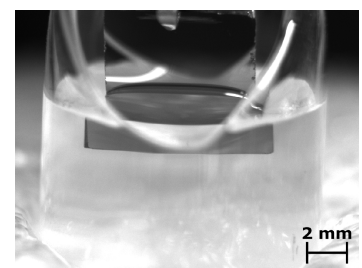

(b)
Fig. 2 (a) Dark-field optical micrograph of a partially immersed AD-MWNT deposition on a Si chip. The visible sharp boundary separates the untouched deposition on the upper half and the immersed region where the liquid contact line has swept away most of the nanotube material. (b) View of the immersion process, captured by a CCD camera.

samples with two samples per each velocity. A scripted ImageJ batch analysis tool was developed for the identification of tubular species in the data, to tabulate the fraction of MWNTs of all deposited particles.

Raman spectroscopy was performed on individual MWNTs in the samples with a reference grid. From these, 6 MWNTs were selected that did not have any amorphous carbon debris in their vicinity. The measurements were executed with a home-built Raman setup in a backscattering geometry using $532 \mathrm{~nm}$ excitation wavelength. The beam was focused to the known location of a single MWNT and subsequently collected with a microscope objective. The beam spot size was $\mathrm{c}$. $1 \mu \mathrm{m}$, which enables to focus on a clean MWNT.

Further details of the experiments, imaging and the analysis methodology can be found in the Supplementary information (Online Resource), and in our previous publication [9].

\section{Results}

\subsection{General features of the purification effect}

The immersion treatment results in a massive reduction of particulate deposition throughout the chip. This is illustrated in Figure 2a that shows a dark-field optical microscope image of a chip where the immersion has (exceptionally) been halted before the whole chip has been submerged. The bright matter in the upper segment is deposition which is untouched by the advancing water contact line, and where especially the larger debris particles effectively scatter light. Their nearly complete removal by the immersion process from the lower segment has resulted in a dark appearance. But most significantly, SEM imaging (Fig. 3) shows that clean MWNTs along with some of the smallest debris par-

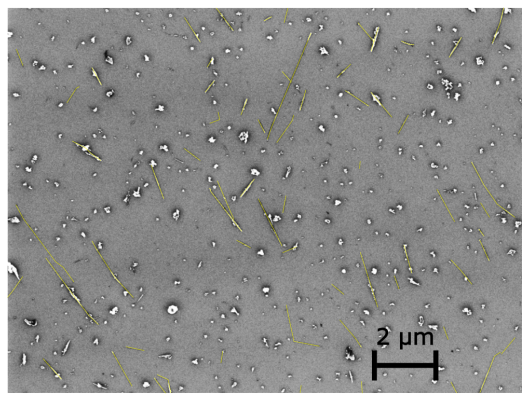

(a)

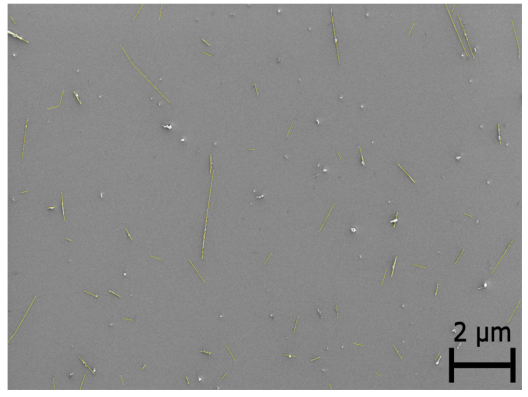

(b)

Fig. 3 SEM micrographs of (a) Spin-coated raw ADMWNT material. (b) A deposition purified with the immersion procedure. The MWNTs were manually emphasized in both images.

ticles remain on the surface throughout the immersed chip. We can thus see that a systematic, strongly selective removal of debris particles over MWNTs occurs in the immersion purification process.

While it is not possible to decouple the advancing and receding contact lines on our present experimental set-up, it is clear that the advancing front was responsible of the purification process. This is convincingly seen from the debris rafts that vacate from the sample in the immersion phase, and subsequently linger on the water surface. It consists of the amorphous carbon nanoparticles and also of MWNTs that happen to be in an adhesive contact with larger debris particles. The floating debris re-attaches during the retraction, unless removed, but no apparent gathering of excess matter is found on the chip in a neatly executed experiment. We conclude that the particles detached from the surface during the immersion have been completely removed from the sample, rather than simply trans-located along its surface. Additional qualitative observations were: Firstly, in separate control experiments, it was verified that on substrates with hydrophobic surface conditions there is no apparent effect on the MWNT deposition, as is expected based on our previous work [9]. Secondly, repeating the immersion process yielded no visible further improvement of the purification effect. 


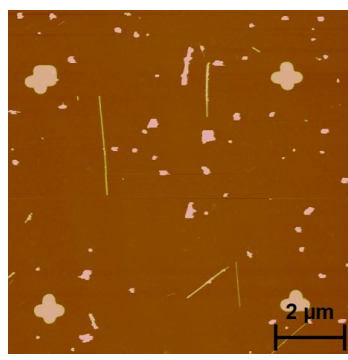

(a)

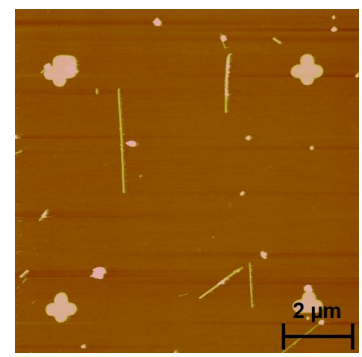

(b)
Fig. 4 An example of a pair of images for quantification of the purification effect: $10 \mu \mathrm{m} \times 10 \mu \mathrm{m}$ AFM frames recorded prior to (a) and after (b) the immersion treatment at the same site.

\subsection{Quantitative estimation of the particle detachment}

AFM images taken on the same location before/after the immersion process (Fig. 4) provide more accurate data on the detachment process. The immersion velocity was varied between the different samples to gauge its effect for the particle detachment. Fig. 5a shows analysis results for the detachment fractions of debris particles and nanotubes. The mean values for the fraction of detached debris and MWNTs are $47 \%$ and $10 \%$, respectively. No apparent velocity dependence is evident for this velocity range. The accompanying error bars for the debris data in Fig. 5a are based on the standard deviation values that were instead calculated framewise, reflecting regional variations in the detachment for a given sample. The numbers of detached MWNTs were too small for meaningful similar estimates. Unfortunately, we could not analyze numerically the size distribution of the debris particles, but it is clear based on the AFM images that, within the size range from $100 \mathrm{~nm}$ to a few $10 \mu \mathrm{m}$, larger particles are detached more frequently than smaller ones. With this observation in mind, the data of Fig. 5a roughly agrees with the impression from figures $2 \mathrm{a}$ and 3 .

We have carefully analyzed the orientation distribution among the detached MWNTs. This is hampered by there already being a certain orientational distribution among the MWNTs, which is a consequence of the spin coating process, known to invoke radial orienta-tion in nanotube depositions [13]. Therefore, detached MWNTs were manually identified, and their orientations inferred from the pre-immersion AFM segmentation data. In figure 5b, are shown MWNT detachment fractions for different orientation angles, with respect to the contact line. The preferential detachment of horizontal MWNTs is clearly evident.

\subsection{Raman spectra}

Figure 6 shows the Raman spectrum of a representative case among the measured samples. The G-band, around $1580 \mathrm{~cm}-1$, also shown in the inset, is strongly prominent while the D-band around $1350 \mathrm{~cm}-1$ is very small or negligible. We could not detect any signal from the low end of the spectrum, where in principle the so called RBM-signal resides. This was, however, expected since the RBM-signal from MWNTs is known to be very difficult to obtain even in ideal conditions, and in our case is likely to be overwhelmed by the scattering from the $\mathrm{Si} / \mathrm{SiO} 2$ substrate.

\section{Discussion}

4.1 Interfacial forces as a factor in particle detachment

Capillary phenomena have to our knowledge not been applied before for the purpose of purifying carbon nanotubes. The key ingredient of the method used in this work is the moving contact line that selectively sweeps away much of the amorphous carbon debris and leaves clean MWNTs on the surface. The surface tension force associated with the liquid interface at the mobile contact line is described with conventional capillary theory. Other possible detachment forces include viscous shear (i.e. drag) forces, gravity, and buoyancy, which are however generally found negligible for small colloids $[12,14]$.

The detachment of spherical microcolloids by the interfacial forces was discussed in the original work by Leenaars \& O'Brien in 1989 [11]. This approach has since been adopted, e.g. by works which have had in mind applications in environmental sciences [15], and, perhaps more relevant to our case, in the intricate cleaning technology of the semiconductor industry [16]. The detachment of MWNTs specifically has also been investigated experimentally [17], but under such conditions that the liquid interface played no role. Another specific topic relevant to our case is that of so called molecu-lar combing $[18,19]$. From these works we can learn of previous attempts to explain the effects of moving liquid (usually water) interfaces on micron-scale particles on substrates. Given the complex nature of the problem, we limit the discussion to our most central results. Among these are the selective removal by the liquid interface of the larger amorphous carbon particles, the dependence of the process on a hydrophilic substrate, and the degree of orientation among the MWNTs left on surface. 


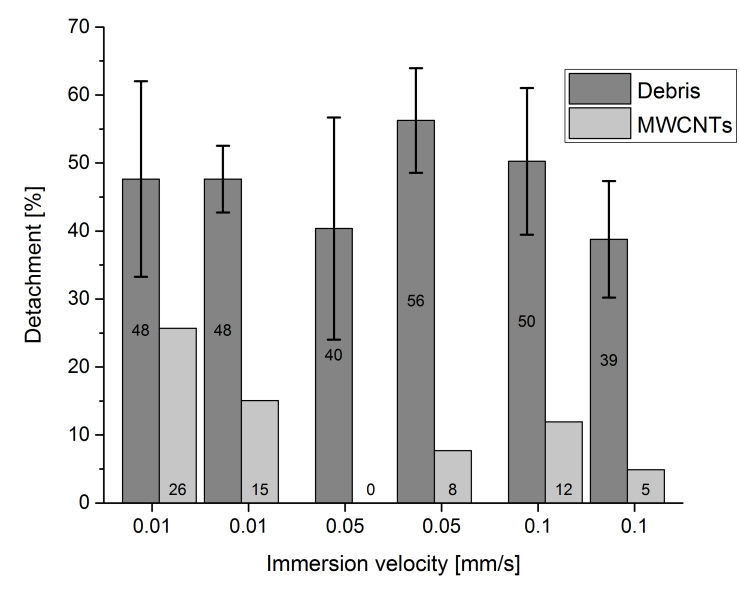

(a)

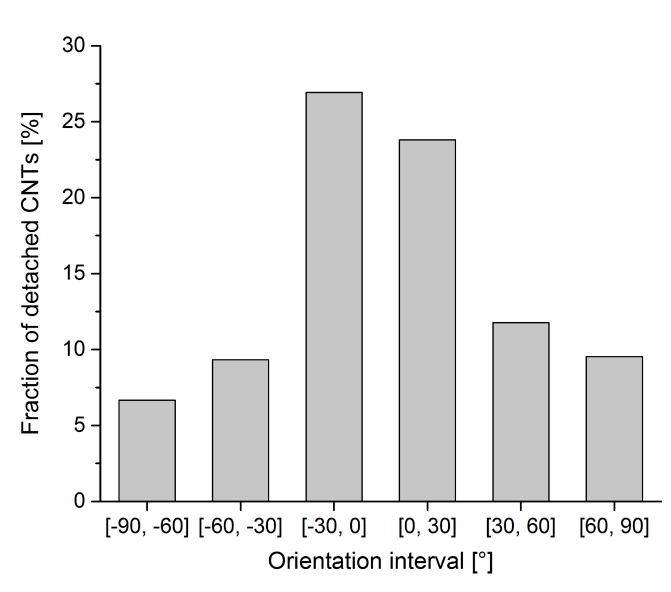

(b)

Fig. 5 (a) Data with error bars showing for amorphous debris particles and MWNTs the fraction of detachment vs. the immersion velocity. The data has been collected from AFM images of the type in Fig. 4. (b) Fraction of MWNTs detached from the samples with respect to their orientation. Preferential detachment of horizontal tubes $\left(0^{\circ}\right.$ angle), i.e. parallel to the propagating contact line, is implied.

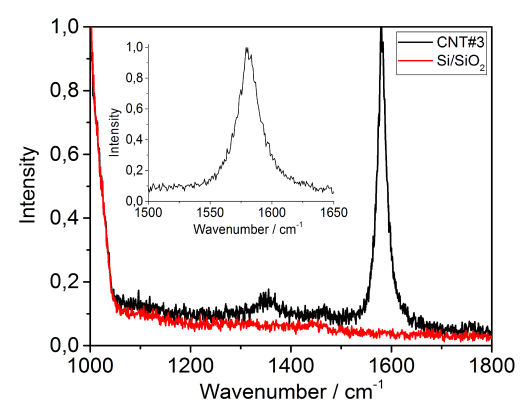

Fig. 6 Raman spectrum of a representative case among the measured MWNTs. The signal from pure substrate $(\mathrm{Si} / \mathrm{SiO} 2)$ is also shown. Inset: close-up view of the Gband region.

\subsection{The surface tension force at the contact line}

Before presenting the model for the interfacial force, we mention briefly a few factors that are such that we by necessity, as just mentioned above, simplify in order to concentrate on the central results. One is the adhesion of the MWNTs and the amorphous carbon particles. Both of these are hydrophobic and thus should be only weakly adhering to the hydrophilic substrate. We assume that this is one reason for the decisive difference in the process between the behaviors of the hydrophilic and hydrophobic surfaces. Other factors are the substrate-liquid contact angle $\alpha$ and the colloidliquid contact angle $\theta$. Their direct utilization in the force calculations is associated with a degree of uncertainty, given that we are unable to verify the validity of these concepts in the nanometer scale of individual MWNTs. Nevertheless, we apply existing analytical methods to our situation, as we believe that they approximately address the essential features of the purification process.

Figure 7a depicts schematically the physical scenario that is central to our analysis in a simplified spherical geometry: A propagating liquid interface passes over a colloid particle of radius $R$ resting on a surface. Unless the particle itself moves as the liquid interface immerses it, the resulting surface tension force on the particle $F_{\gamma}$ will continuously change direction and magnitude. The maxima of its vertical and lateral components are expressed as [14]:

$$
\begin{aligned}
& F_{\gamma, \max }^{z}=2 \pi R \gamma \sin ^{2}\left(\frac{\theta}{2}\right) \cos \alpha \\
& F_{\gamma, \max }^{x, y}=2 \pi R \gamma \sin ^{2}\left(\frac{\theta}{2}\right) \sin \alpha
\end{aligned}
$$

where $\gamma$ is the surface tension. The vertical component $F_{\gamma, \max }^{z}$ is generally considered the detachment force that works against adhesion forces, whereas the lateral component $F_{\gamma}^{x, y}$ max is taken to contribute to the translocation of the colloids along the surface. The experiments of this work indicate almost solely vertical detachment takes place rather than translocation.

Qualitatively, eq. 1 helps to explain a number of the observed effects in our process. Firstly, the force scales linearly with $\mathrm{R}$, while the adhesion force, assumed to be largely dependent on the contact area between the particles and the surface, increases much more slowly with 


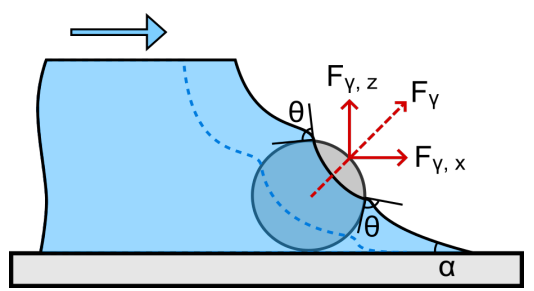

(a)

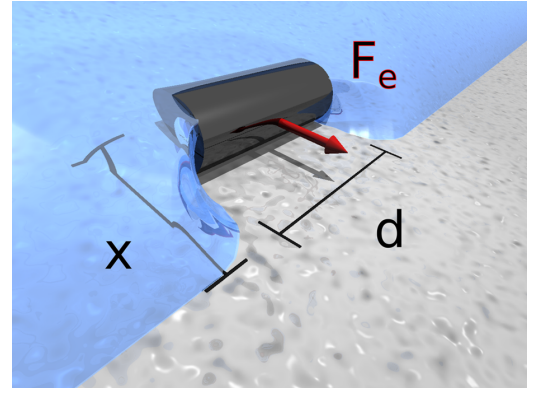

(b)

Fig. 7 (a) Cross-sectional scheme of a liquid interface at the contact line interacting with a spherical colloid through the surface tension force $F_{\gamma}$. The progression of the interface, from left to right, is represented as the blue dashed line and the black solid line. (b) 3D view of an advancing contact line pinning onto a single defect (i.e. carbon nanotube). The deformation of the contact line by $x$ gives rise to an elastic shear force $F_{\mathrm{e}}$.

R. Then the selective removal of larger amorphous carbon particles is understandable. On the other hand, a clean nanotube can have a larger contact area/volume ratio that enhances their adhesion to the surface relative to the irregularly shaped debris particles. This reasoning is then consistent with the observed purification effect of the immersion process. Secondly, the force is maximum for maximal $\theta$ and minimal $\alpha$, corresponding to the contact angles to hydrophobic particles and hydrophilic substrates, respectively. Our pristine MWNTs should be highly hydrophobic, as are the amorphous carbon nanoparticles, and we have a hydrophilically treated Si surface. The wetting property of the substrate thus provides an additional factor besides the adhesion mentioned above, whereby the hydrophilicity of the surface enables the liquid interface to remove the debris particles.

To estimate the magnitude of the force on debris particles, we consider them as spheres with radii commonly ranging from $10 \mathrm{~nm}$ to $1 \mu \mathrm{m}$. The contact angle $\theta$ is taken as that of a water droplet on graphite $[20,21]$, approx. $90^{\circ}$. Inserting $\gamma \approx 73 \mathrm{mN} / \mathrm{m}$ and $\alpha \approx 0^{\circ}$

for hydrophilic Si, we acquire from eq. 1 that $F_{\gamma, \text { max }}^{z} \approx$ $0.23 \mathrm{~N} / \mathrm{m} \cdot R$. This gives for the magnitude of the verti- cally directed detachment force of a few $\mathrm{nN}$ for a $10 \mathrm{~nm}$ particle.

The surface tension force of eq. 1 does not take into account the possibility that the colloidal particle, if strongly adhered to the surface, also locally inhibits the free motion of the liquid interface, causing a deformation $x$ in its profile, as pictured in Fig. 7b. This tension gives rise to an additional elastic deformation force experienced by the colloidal particle. The deformation of the liquid interface due to surface pinning sites was first described by Joanny \& de Gennes in 1984 in their work on contact angle hysteresis [22]. The force is given as [19]:

$F_{\mathrm{e}} \approx \frac{\pi \gamma \alpha^{2}}{\log L / d} \cdot x$

Here $L$ is a macroscopic scale cut-off distance, and $d$ the characteristic size of the particle. Thus this force has a very different size dependence compared to the previous one. Particles with rough texture or jagged edges allow for most prolonged pinning, as demonstrated in Ref. [23]. The elastic force could therefore be one of the factors promoting the selective removal of debris in favor of carbon nanotubes given the irregular shape of the debris particles.

The standard equations apply to spherical particles. It is intuitively obvious that the MWNT orientation with respect to the contact line can enormously modify the force. If the tube axis is parallel to the contact line, the force can be up to three orders of magnitude larger than in the perpendicular case. We therefore present that the orientation-selective removal of MWNTs of Fig. 5b is explained by modification of $F_{\gamma}$ and $F_{\mathrm{e}}$ by geometrical factors. A comparable situation has been studied, and the effect verified, with ellipsoidal polystyrene colloidal particles in Ref. [24], though with a much smaller aspect ratio than with MWNTs. The orientation effect is also in accordance with our previous study [9]. However, the effect is much smaller than would be implied by the aspect ratio of AD-MWNTs. This is understandable, considering that in our experimental conditions, the macroscopic contact line is unlikely to be homogenously oriented at the micrometer scale.

In the existing literature, the elastic deformation forces (eq. 3) have been considered as a source of torque that aligns or reorientates $1 \mathrm{D}$ nano-objects, e.g. DNA $[18,25]$, in a process referred as molecular combing. Systematic reorientation of SWNTs by mobile liquid interfaces has been previously reported [19], and more recently, for SWNT-DNA complexes [26]. In these cases, the interface applied in the process was however that 
of the receding, particle-containing solution, which is in contrast to our case, where the advancing contact line of pure water is utilized.

We have thus shown that by examining the features of eqs. 1 and 3, together with the assumption of weak adherence of the hydrophobic AD-MWNT material to the hydrophilic substrate, we can qualitatively explain the central effects of the immersion process that results in the purification of the AD-MWNT material. Next we demonstrate via two examples how these findings can be utilized.

\subsection{Raman spectra of individual MWNTs}

The Raman spectra of individual AD-MWNTs have been investigated only in a few works [27-29], and in these the number of MWNTs measured and reported is small. The high crystallinity of AD-MWNTs should show up in the Raman spectra as a weak D-band, which is associated with disorder in graphitic materials $[1,30]$. In a nice work on Raman spectroscopy of bulk CVD MWNT material [31], it was shown that a high temperature treatment $\left(2800^{\circ} \mathrm{C}\right)$ changes the disordered structure of the MWNTs into highly crystalline form, as directly observed in TEM. The change is seen in the Raman spectra as a drastic reduction of the D-band.

In our work, we observe that the D-band intensity is negligible compared to the G-band in all the measured tubes. This result thus demonstrates that our purification process in a relatively simple manner can separate the AD-MWNTs from the amorphous carbon debris and that the tubes are of a high quality. The $\mathrm{G}$ band in most of the samples, such as in Fig. 6, can be fitted by a single Lorentzian function. However, we also observed elevated $\mathrm{G}$ band frequencies and a splitted $\mathrm{G}$ band. The AD-MWNT is expected to exhibit this kind of rich features in its Raman spectra due to the presence of several concentric layers, which can be further investigated once clean samples can be easily produced.

\subsection{Utilization of the method: Suspended MWNTs}

To further highlight the scope of our cleaning method, we present results of the immersion purification treatment applied on MWNT samples deposited on substrates with etched trench-like groove patterns. Such structures are of importance e.g. in nanomechanical devices based on suspended MWNTs.

The patterned samples utilized in this experiment were fabricated separately on $\mathrm{Si} / \mathrm{SiO}_{2}$ substrates via ebeam lithography. Details of the process are given in the Online Resource. When utilized as substrates for

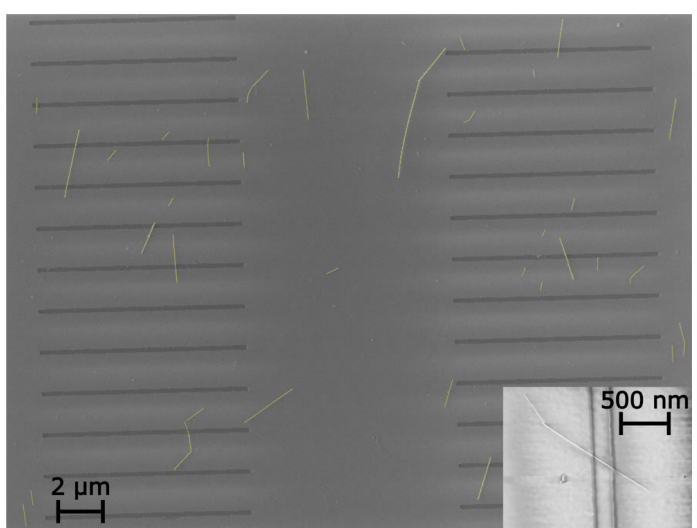

Fig. 8 SEM image of a pair of groove columns, showing the cleaned deposition. MWNTs were manually highlighted for clarity. Numerous instances of tube crossings over the troughs can be seen. The inset shows an individual crossing event in detail.

MWNTs, all the purification steps were then carried out as for the regular samples. The grooves were oriented parallel to the liquid surface during the immersion to allow for maximal number of crossover incidents of the MWNTs. Inspection under dark-field optical microscope revealed that the presence of the trenches does not appear to inhibit the purification effect. SEM imaging (fig. 8) of the patterned region shows a remarkably clean deposition of MWNTs, with several individual tubes spanning across the trenches as desired.

The Raman experiment and the suspended tubes are examples of the most immediate ways to utilize our cleaning method, namely for performing single-tube experiments. The method makes in a simple way available on a single chip a large number of clean high quality AD-MWNTs. Previous work on AD-MWNTs has often been based on results from very few tubes, due to the difficulties inherent with raw AD-MWNT material. This progress also bring to the domain of ADMWNTs some of the possibilities that have been in use among SWNTs for more than decade, where CVD growth of SWNT on surfaces [32] produces clean high quality SWNTs. The purified depositions consist of isolated, relatively clean AD-MWNTs with some remaining amorphous carbon particles sparsely dispersed inbetween. A further development of the process would be to increase the density of MWNTs so that they overlap over macroscopic distances. This would create a $2 \mathrm{D}$ network of high quality AD-MWNTs and thereby to add new possibilities to the present carbon nanotube thin film technology. 


\section{Conclusions}

We have shown that application of a mobile liquid interface provides an efficient and simple method for purification of AD-MWNT material spin coated on hydrophilic silicon substrates. The purification effect is attributed to the capillary forces that selectively remove amorphous carbon particles much more efficiently than the MWNTs. A dedicated image analysis and segmentation tool was developed for identifying MWNTs from AFM data for the purposes of quantitative analysis of the process. This revealed the (expected) tendency of the liquid interface to interact more with tubes oriented parallel to the contact line. We demonstrated the utility of the purification process by preparing large numbers of suspended clean individual AD-MWNTs on a Si substrate.

The purification process has an advantage over most CNT purification procedures in that it does not cause any mechanical damage or chemical modification to the tubes. This was seen from Raman spectra of individual MWNTs, where the negligible D-band signal proved their high quality. A disadvantage of the method is that it is presently bound to surfaces and does not produce macroscopic amounts of purified tubes. It is conceivable though, that these boundary conditions can be overcome, and moreover, we envision that the present technique can be developed to yield $2 \mathrm{D}$ networks of purified AD-MWNTs. The most immediate outcome of the method, though, is that it opens new prospects for performing such experiments that need an easy availability of clean high quality single MWNTs.

Acknowledgements M. Sc. Hannu Pasanen is acknowledged for his help with the preliminary experiments, and M. Sc. Roope Lehto and Prof. Jussi Timonen for useful discussions. Dr. Sami Malola is acknowledged for producing the 3D illustration in Fig. 7b. Author Matti J. Hokkanen gratefully acknowledges financial support from the Finnish National Graduate School in Material Physics (NGSMP) and the National Doctoral Programme in Nanoscience (NGS-NANO).

Conflict of interest: the authors declare that they have no conflict of interest.

\section{References}

1. J. H. Lehman, M. Terrones, E. Mansfield, K.E. Hurst, V. Meunier, Carbon 49, 8 (2011).

2. M. Buitelaar, A. Bachtold, T. Nussbaumer, M. Iqbal, C. Schonenberger, Phys. Rev. Lett. 88, 15 (2002).

3. D. Mtsuko, A. Koshio, M. Yudasaka, S. Iijima, M. Ahlskog, Phys. Rev. B 91, 19 (2015).
4 R. Tarkiainen, M. Ahlskog, A. Zyuzin, P. Hakonen, M. Paalanen, Phys. Rev. B 69, 3 (2004).

5. H. Jackman, P. Krakhmalev, K. Svensson, J. Appl. Phys. 117, 8 (2015).

6. J. Cumings, A. Zettl, Phys. Rev. Lett. 93, 8 (2004).

7. X. Wang, Y. Ouyang, L. Jiao, H. Wang, L. Xie, J. Wu, J. Guo, H. Dai, Nat. Nanotechnol. 6, 9 (2011).

8. P.X. Hou, C. Liu, H.M. Cheng, Carbon 46, 15 (2008).

9. M.J. Hokkanen, R. Lehto, J. Takalo, J. Salmela, S. Haavisto, A. Bykov, R. Myllyl, J. Timonen, M. Ahlskog, Colloid. Surface A 482, pp. 624-30 (2015).

10. B. Bhushan, in Springer Handbook of Nanotechnology, 2nd edn, ed. By B. Bhusnan. (Springer, Berlin Heidelberg / New York, 2010) pp. 1387-8.

11. A.F.M. Leenaars, S.B.G O'Brien, Philips J. Res. 44, 2-3 (1989).

12. P. Sharma, M. Flury, J. Zhou, J. Colloid. Interf. Sci. 326, 1 (2008).

13. M.C. LeMieux, M. Roberts, S. Barman, Y.W. Jin, J.M. Kim, Z. Bao, Science 321, 5885 (2008).

14. S. Aramrak, M. Flury, J.B. Harsh, Langmuir 27, 16 (2011).

15. V. Lazouskaya, L.P. Wang, D. Or, G. Wang, J.L. Caplan, Y. Jin, J. Colloid. Interf. Sci. 406, pp. 44-50 (2013).

16. G.W. Gale, R.J. Small, K.A. Reinhardt, in Handbook of silicon wafer cleaning technolog, 2nd edn, ed. by K.A. Reinhardt, W. Kern (William Andrew Inc., Norwich NY, 2008), p. 307.

17. P. Yi, K.L. Chen, Environ. Sci. Technol. 47, 21 (2013).

18. A. Bensimon, A.J. Simon, A. Chiffaudel, V. Croquette, F. Heslot, D. Bensimon, Science 265, 5181 (1994).

19. S. Gerdes, T. Ondarcuhu, S. Cholet, C. Joachim, Europhys. Lett. 48, 3 (1999).

20. Y.J. Shin, Y. Wang, H. Huang, G. Kalon, A.T.S. Wee, Z. Shen, C.S. Bhatia, H. Yang, Langmuir 26, 6 (2010).

21. J. Rafiee, X. Mi, H. Gullapalli, A.V. Thomas, F. Yavari, Y. Shi, P.M. Ajayan, N.A. Koratkar, Nat. Mater. 11, 3 (2012).

22. J.F. Joanny, P.G. de Gennes, J. Chem. Phys. 81, 1 (1984).

23. N. Chatterjee, M. Flury, Langmuir 29, 25 (2013).

24. S. Aramrak, M. Flury, J.B. Harsh, R.L. Zollards, H.P. Davis, Langmuir 29, 19 (2013).

25. D. Bensimon, A.J. Simon, V. Croquette, A. Bensimon, Phys. Rev. Lett. 74, 23 (1995).

26. C.Y. Khripin, M. Zheng, A. Jagota, J. Colloid. Interf. Sci. 330, 2 (2009).

27. X. Zhao, Y. Ando, L.C. Qin, H. Kataura, Y. Maniwa, R. Saito, Appl. Phys. Lett. 81, 41 (2002).

28. S. Nanot, M. Millot, B. Raquet, J.M. Broto, A. Margrez, J. Gonzales, Physica E 42, 9 (2010).

29. X. Hou, L. Sheng, L. Yu, K. An, Y. Ando, X. Zhao, J. Raman Spectrosc. 43, 10 (2012).

30. M.S. Dresselhaus, A. Jorio, R. Saito, Annu. Rev. Condens. Matter Phys. 1, pp. 89-108 (2010).

31. S.N. Bokova, E.D. Obraztsova, V.V. Grebenyukov, K.V. Elumeeva, A.V. Ishchenko, V.L. Kuznetsov, Phys. Status Solidi B 247, 11-12 (2010).

32. Y. Chen, Y Zhang, Y. Hu, L. Kang, S. Zhang, H. Xie, D. Liu, Q. Zhao, Q. Li, J. Zhang, Adv. Mater. 26, 34 (2014). 